\title{
The Yang-Mills deconfinement transition from a high temperature expansion
}

\author{
Jangho Kim, Anh Quang Pham*, Owe Philipsen, Jonas Scheunert \\ Institut für Theoretische Physik, Goethe-Universität Frankfurt, \\ Max-von-Laue-Str. 1, 60438 Frankfurt am Main, Germany \\ E-mail: jkim, pham, philipsen, scheunert@th.physik.uni-frankfurt.de
}

\begin{abstract}
The high temperature expansion is an analytical tool to study critical phenomena in statistical mechanics. We apply this method to 3 d effective theories of Polyakov loops, which have been derived from 4d lattice Yang-Mills by means of resummed strong coupling expansions. In particular, the Polyakov loop susceptibility is computed as a power series in the effective couplings. A Padé analysis then provides the location of the phase transition in the effective theory, which can be mapped back to the parameters of $4 \mathrm{~d}$ Yang-Mills. Our purely analytical results for the critical couplings $\beta_{c}\left(N_{\tau}\right)$ agree to better than $10 \%$ with those from Monte Carlo simulations. For the case of $S U(2)$, also the critical exponent $\gamma$ is predicted accurately, while a first-order nature as for $S U(3)$ cannot be identified by a Padé analysis. The method can be generalised to include fermions and finite density.
\end{abstract}

37th International Symposium on Lattice Field Theory - Lattice2019

16-22 June 2019

Wuhan, China

${ }^{*}$ Speaker. 


\section{Introduction}

A lot of effort is dedicated to studying the phase diagram of strongly interacting matter under extreme conditions, which plays an important role for nuclear astrophysics, particle physics and heavy ion collisions. Unfortunately, at finite $\mu$ all Monte Carlo algorithms suffer from the "fermion sign problem." Approximate methods work only for small chemical potential $\mu=\mu_{B} / 3 \leq T$ [1], for which there is no sign of a critical point. Based on stochastic quantization methods, complex Langevin simulations do not have a sign problem, but for complex actions the correct results are not guaranteed [2].

This motivates the development of effective theories. On one hand, with part of the degrees of freedom integrated out, the sign problem of effective theories in the cold and dense region of QCD is weaker. Thus, it is possible to simulate with standard Monte Carlo or complex Langevin. On the other hand, one can use series expansion methods to carry out analytic computations [3], for which the sign problem is irrelevant. In this paper, we present a scheme known as "high temperature expansion" in statistical mechanics, which is also applicable to the effective theories of interest. As a first test, we apply it to Yang-Mills theory, where we can compare with full simulation results.

\section{Effective theories for Yang-Mills}

We start with reviewing the effective theories. Let us consider the $S U\left(N_{c}\right)$ Wilson action which is defined to be

$$
S(U)=\frac{\beta}{2 N_{c}} \sum_{P}\left(\operatorname{Tr} U_{P}+\operatorname{Tr} U_{P}^{\dagger}\right)=\sum_{P} S_{P}
$$

where the coupling is $\beta=2 N_{c} / g^{2}$, the sum runs over all plaquettes on the lattice and

$$
U_{P}(x)=U_{\mu}(x) U_{\nu}(x+\hat{\mu}) U_{\mu}(x+\hat{\nu})^{\dagger} U_{\nu}(x)^{\dagger} .
$$

The associated partition function is given by

$$
Z=\int \mathcal{D} U \exp [S(U)]
$$

where $\mathcal{D} U=\prod_{x, \mu} \mathrm{d} U_{\mu}(x)$ is the measure and $\mathrm{d} U_{\mu}(x)$ is the Haar measure on $S U\left(N_{c}\right)$.

We obtain a three-dimensional effective theory via integrating out all spatial links $U_{i}(x)$, which was first done for $S U(2)$ YM theory [4],

$$
\begin{aligned}
Z & =\int \mathcal{D} U_{0} \exp \left[S_{\text {eff }}\left(U_{0}\right)\right], \\
S_{\text {eff }}\left(U_{0}\right) & =\ln \int \mathcal{D} U_{i} \exp [S(U)]=\lambda_{1} S_{1}+\lambda_{2} S_{2}+\ldots
\end{aligned}
$$

Here $\lambda_{n}\left(N_{\tau}, \beta\right)$ denote the effective couplings, which are functions of the temporal extent $N_{\tau}$ and the original coupling $\beta$. The $S_{i}$ depend only on the temporal links $U_{0}(x)$ and for $N_{c} \leq 3$ the dependency can be re-expressed in terms of Polyakov loops

$$
L_{\vec{x}}=\operatorname{Tr} W_{\vec{x}}=\operatorname{Tr} \prod_{\tau=0}^{N_{\tau}-1} U_{0}(\tau, \vec{x}) .
$$


In practice, the effective action is obtained by a character expansion of the gaugeinvariant function $\exp \left(S_{P}\right)$, and for the orders considered here the integrations over the spatial links can be done using the orthogonality relations of characters. (For details on the character expansion, see [5]). This results in a power series in the coefficient of the fundamental character, $u(\beta)$, which is always smaller than one for finite $\beta$.

Here we work with the nearest neighbour effective action derived in [6] which includes a logarithmic resummation and reads (since we only deal with one coupling we drop the index on $\lambda_{1}$ from now on)

$$
S_{\mathrm{eff}}= \begin{cases}\sum_{\langle\vec{x}, \vec{y}\rangle} \ln \left[1+\lambda\left(N_{\tau}, u\right) L_{\vec{x}} L_{\vec{y}}\right], & \text { for } N_{c}=2, \\ \sum_{\langle\vec{x}, \vec{y}\rangle} \ln \left[1+\lambda\left(N_{\tau}, u\right)\left(L_{\vec{x}} L_{\vec{y}}^{*}+L_{\vec{x}}^{*} L_{\vec{y}}\right)\right], & \text { for } N_{c}=3\end{cases}
$$

with the effective coupling

$$
\begin{aligned}
\lambda\left(u, N_{\tau} \geq 5\right)=u^{N_{\tau}} \exp \left[N_{\tau}\left(4 u^{4}-4 u^{6}+\frac{140}{3} u^{8}-\frac{36044}{405} u^{10}\right)\right], & \text { for } N_{c}=2, \\
\lambda\left(u, N_{\tau} \geq 6\right)=u^{N_{\tau}} \exp \left[N _ { \tau } \left(4 u^{4}+12 u^{5}-14 u^{6}-36 u^{7}+\right.\right. & \text { for } N_{c}=3 . \\
\left.\left.\frac{295}{2} u^{8}+\frac{1851}{10} u^{9}+\frac{1055797}{5120} u^{10}\right)\right], &
\end{aligned}
$$

\section{High temperature expansion for the effective YM theory}

To solve the effective theories, we used high temperature expansions ${ }^{1}$ which played an essential role for studying critical phenomena before the Monte-Carlo method. General concepts of the high temperature expansion applied to Ising models can be found, for instance, in [7]. This method is particularly simple for Ising models because spins can only take two values +1 or -1 , but for effective gauge theories it is more complicated when our "spins" are Polyakov loops.

Let us focus on the improved effective action (2.7) for $N_{c}=3$, the $N_{c}=2$ can be treated analogously with the simplification that the Polyakov loop is always real. Our main goal is to extract information about phase transitions from the series. Thus, we are interested in the Polyakov loop susceptibility $\chi_{L}$ which peaks near phase transitions

$$
\chi_{L}=\left.\frac{1}{V} \frac{\partial^{2}}{\partial J^{2}} \ln Z[J]\right|_{J=0}
$$

where $Z[J]$ is the partition function coupled to an external source $J$,

$$
Z[J]=\int \mathcal{D} \mathbb{U}[J] \prod_{\langle\vec{x}, \vec{y}\rangle}\left[1+\lambda\left(L_{\vec{x}} L_{\vec{y}}^{*}+L_{\vec{x}}^{*} L_{\vec{y}}\right)\right]=\int \prod_{\vec{x}} \mathrm{~d} W_{\vec{x}} e^{J\left(L_{\vec{x}}+L_{\vec{x}}^{*}\right)} \prod_{\langle\vec{x}, \vec{y}\rangle}\left[1+\lambda\left(L_{\vec{x}} L_{\vec{y}}^{*}+L_{\vec{x}}^{*} L_{\vec{y}}\right)\right] .
$$

\footnotetext{
${ }^{1}$ High temperature expansion is the name adapted from statistical mechanics, in context of effective theories we expand our theories around small couplings $\lambda$.
} 
We expand the partition function in the effective coupling $\lambda$,

$$
\begin{aligned}
Z[J]= & \int \mathcal{D} \mathbb{U}[J]+\lambda \int \mathcal{D} \mathbb{U}[J] \sum_{\langle\vec{x}, \vec{y}\rangle} 2 \operatorname{Re}\left(L_{\vec{x}} L_{\vec{y}}^{*}\right) \\
& +\lambda^{2} \int \mathcal{D} \mathbb{U}[J] \sum_{\langle\vec{w}, \vec{x}\rangle} \sum_{\langle\vec{y}, \vec{z}\rangle} 4 \operatorname{Re}\left(L_{\vec{w}} L_{\vec{x}}^{*}\right) \operatorname{Re}\left(L_{\vec{y}} L_{\vec{z}}^{*}\right)+\mathcal{O}\left(\lambda^{3}\right) .
\end{aligned}
$$

Fortunately, instead of lengthy and complicated expressions there is an elegant graph representation for (3.2),

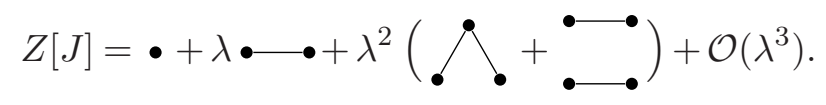

Here the order of $\lambda$ corresponds to the number of bonds of an associated graph, and from the number of bonds coupled to a vertex one can count the number of Polyakov loops at this vertex. Note that for our effective theories we need to include open graphs, for instance at $\lambda^{2}$, while those contributions drop out in most cases for Ising models. There are two main computations to be performed:

- Graph counting. In general, each graph has its own embedding number, which gives the number of ways this graph appears on the lattice. For a detailed discussion we refer to [7]. On a three dimensional cubic lattice the embedding numbers of each graph in (3.3) are

$$
V, \quad 3 V, \quad 15 V, \quad 9 V^{2} / 2-33 V / 2
$$

where $V=N_{s}^{3}$ is the number of lattice sites.

- Graph evaluation. As an example, the fist order graph of $Z[J]$ is calculated explicitly as follows

$$
\begin{aligned}
\bullet \bullet & =\int \mathcal{D} \mathbb{U}[J] \sum_{\langle\vec{x}, \vec{y}\rangle} 2 \operatorname{Re}\left(L_{\vec{x}} L_{\vec{y}}^{*}\right) \\
& =3 V\left(\int \mathrm{d} \mathbb{U}[J]\right)^{V-2} 2\left(\int \mathrm{d} \mathbb{U}[J] L\right)\left(\int \mathrm{d} \mathbb{U}[J] L^{*}\right),
\end{aligned}
$$

The nearest neighbor sum gives $3 V$ integrals of the same kind, i.e., it reduces our computations to single site $S U\left(N_{c}\right)$ integrals as one can see in the second equation. Finally, it is easy to compute $\chi_{L}$ with use of (3.1) and the group integrals at the sites

$$
I(n, m)=\int_{S U\left(N_{c}\right)} \mathrm{d} W L^{n} L^{* m},
$$

can be evaluated for $N_{c}=3$ using the formula given in [8]. This formula is based on a generating functional derived in [9]. This reference also contains a generating functional for the $S U(2)$ case, which one can handle a similar fashion.

It is obvious that beyond the first few orders the computations are cumbersome, but the graphs can be generated, embedded and evaluated by a computer. The original algorithm to compute the embedding constants was developed by Martin [10], here we use the code provided in [11]. 


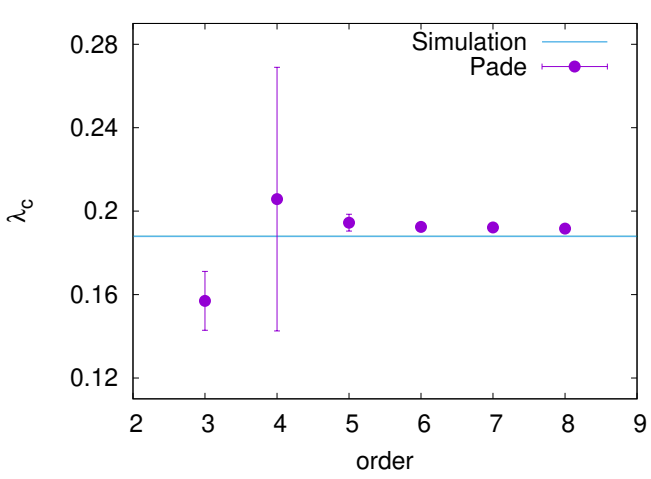

(a) $S U(3)$

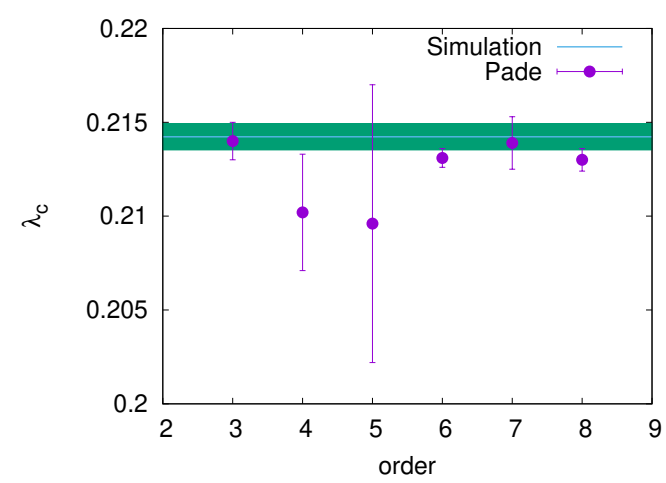

(b) $S U(2)$

Figure 1: Critical coupling $\lambda_{c}$ to different orders

\section{Series analysis and results}

Phase transitions are signalled by non-analyticities in the susceptibility. In particular, near a critical point the susceptibility diverges with a critical exponent, such that its logderivative has a simple pole,

$$
\chi_{L} \sim \frac{1}{\left(\lambda-\lambda_{c}\right)^{\gamma}} \quad \longrightarrow \quad \frac{\mathrm{d}}{\mathrm{d} \lambda} \ln \chi_{L} \sim \frac{-\gamma}{\lambda-\lambda_{c}} .
$$

At a first-order phase transition, instead, there is a discontinuity at the critical coupling. The generic expression obtained by expansion methods is a finite power series in $\lambda$,

$$
\chi_{L}=\sum_{n=0}^{N+1} s_{n} \lambda^{n},
$$

which cannot show such behaviour.

A standard method to extract information about criticality from the available coefficients is the Padé analysis [12]. Finite series can be extended to infinite order by constructing ratios of two polynomials of degrees $L, M$, whose expansion agrees with the original series to its given order $N$. We then approximate

$$
\frac{\mathrm{d}}{\mathrm{d} \lambda} \ln \chi_{L} \sim[L / M]=P(\lambda) / Q(\lambda)=\frac{p_{0}+p_{1} \lambda+\cdots+p_{L} \lambda^{L}}{1+q_{1} \lambda+\cdots+p_{M} \lambda^{M}},
$$

in which the $L+M+1=N+1$ coefficients $p_{0}, p_{1}, \ldots, p_{L}, q_{1}, \ldots, q_{M}$ are determined by equating like powers of $\lambda$. Diagonal Padé approximants are expected to be the most stable ones, since they are invariant under Möbius transformations and thus under resummations of the series. We extract our results from the table of diagonal and near-diagonal approximants $[M / M],[M-1, M+1]$ and $[M+1 / M-1]$. At order $\lambda^{N}$ in (4.4) we take either three or two approximants depending on whether $N$ is even or odd, e.g. , ([1/2], [2/1]) at order 3 and $([1 / 3],[2 / 2],[3 / 1])$ at order 4 . For each of these we determine the poles in $\lambda$ and their residues. We then compute the mean value and quote as systematic error the maximum deviation between pairings $\left|\lambda_{j}-\lambda_{i}\right|$, where $i$ labels the approximants. 


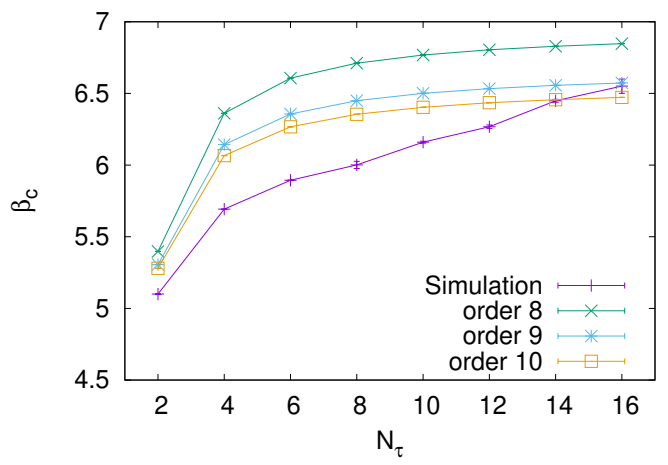

(a)

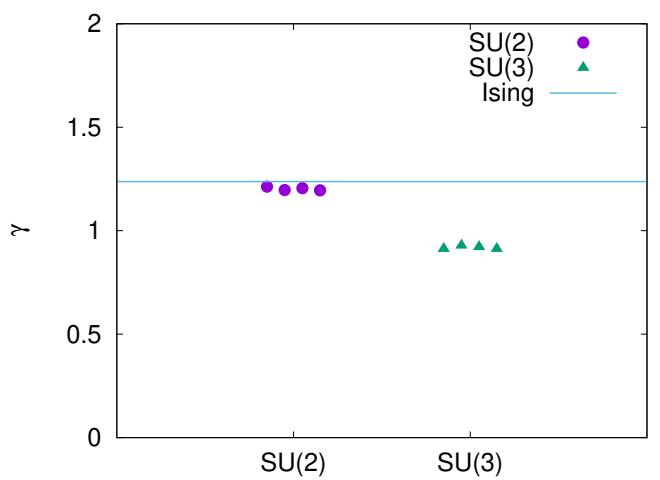

(b)

Figure 2: (a) Critical coupling $\beta_{c}$ by (2.9) for $S U(3)$. (b) Exponent $\gamma$ for $S U(2)$ and $S U(3)$.

Results. Our result for the logarithmic derivative of the Polyakov loop susceptibility up to 8 th order reads

$$
\begin{aligned}
\frac{\mathrm{d}}{\mathrm{d} \lambda} \ln \chi_{L}=6 & +24 \lambda+126 \lambda^{2}+768 \lambda^{3}+3126 \lambda^{4}+20736 \lambda^{5}+87198 \lambda^{6} \\
& +\frac{9489884859334657}{17179869184} \lambda^{7}+\frac{83864630933323767}{34359738368} \lambda^{8}+\ldots
\end{aligned}
$$

Note that the coefficients of (4.4) at order seven and higher become rational due to disconnected contributions. It is now straightforward to estimate the critical coupling $\lambda_{c}$ and the critical exponent $\gamma$ by the Padé analysis. Note however, that Padé approximants always produce poles, even in the case of a first-order transition. In this case they signal the end of the metastability region and thus are an upper bound on the true critical coupling. For an example, see the $q$ state Potts model [13].

In Figure 1(a) we show the critical coupling $\lambda_{c}$ for $N_{c}=3$ to each order of (4.4). The best estimate of $\lambda$ is within $2 \%$ of the simulation result for that model [6]. Note that, even though the results have stabilised with inreasing order, they slightly overshoot the true value. This is due to the first-order nature of the transition, where the series probes the end of the metastability region. The same computations for the case $N_{c}=2$ are shown in Figure 1(b). Here the best estimate for $N_{c}=2$ is within $1 \%$ from simulation data.

We can now invert (2.9) to extract the critical couplings $\beta_{c}$ and compare with results of the full four-dimensional Yang-Mills theory [14]. Figure 2(a) shows this for $S U(3)$ and we observe a satisfactory convergence behavior of strong coupling series to the full result.

In Figure 2(b), by using four different Padé approximants [4/4], [4/3], [3/3], and [3/5] we show the resulting critical exponents $\gamma$ of our effective theory. The estimates for $S U(2)$ accurately reproduce the value for the universality class of the $3 d$ Ising model, while the $S U(3)$ case gives values different from all known universality classes. This is consistent with its nature as a first order phase transition, see the discussion above.

\section{Conclusions}

We have considered a $3 \mathrm{~d}$ effective theory for finite temperature $S U(2), S U(3)$ YangMills in 4d, obtained by strong coupling methods, which only depends on Polyakov loops. 
We computed the Polyakov loop susceptibility as a series in the effective coupling and, by means of a Padé analysis, extracted critical couplings $\beta_{c}$ for the phase transition by purely analytical methods. The results agree to better than $10 \%$ with simulations for $N_{\tau}=2-16$. For $S U(2)$, also the critical exponent $\gamma$ is accurately reproduced, while Padé analyses generally cannot identify a first-order transition. This work demonstrates the benefit of going via an effective theory, compared to a strong coupling expansion of the full theory [15], and can be straightforwardly extended to include fermions at finite density.

\section{Acknowledgments}

This work is supported by the Deutsche Forschungsgemeinschaft (DFG) through the grant CRC-TR 211 "Strong-interaction matter under extreme conditions" and by the Helmholtz International Center for FAIR within the LOEWE program of the State of Hesse.

\section{References}

[1] P. de Forcrand PoS LAT2009 (2009) 010, [1005.0539].

[2] G. Aarts, F. A. James, E. Seiler, and I.-O. Stamatescu Eur. Phys. J. C71 (2011) 1756, [1101.3270].

[3] J. Glesaaen, M. Neuman, and O. Philipsen JHEP 03 (2016) 100, [1512.05195].

[4] J. Polonyi and K. Szlachanyi Phys. Lett. 110B (1982) 395-398.

[5] J.-M. Drouffe and J.-B. Zuber Phys. Rept. 102 (1983) 1.

[6] J. Langelage, S. Lottini, and O. Philipsen JHEP 02 (2011) 057, [1010.0951]. [Erratum: JHEP07,014(2011)].

[7] C. Domb and M. S. Green, eds., Phase Transitions and Critical Phenomena, vol. 3. Academic Press Inc. (London) Ltd., 1974.

[8] C. Gattringer Nuclear Physics B 850 (2011), no. 2242 - 252.

[9] S. Uhlmann, R. Meinel, and A. Wipf J. Phys. A40 (2007) 4367-4390, [hep-th/0611170].

[10] J. L. Martin in Phase Transitions and Critical Phenomena, vol. 3, ch. 2, pp. 97-112. Academic Press Inc. (London) Ltd., 1974.

[11] J. Oitmaa, C. Hamer, and W. Zheng, Series expansion methods for strongly interacting lattice models. Cambridge University Press, 2011.

[12] A. J. Guttmann in Phase Transitions and Critical Phenomena, vol. 13, ch. 1, pp. 1-234. Academic Press Inc. (London) Ltd., 1989.

[13] M. Hellmund and W. Janke Phys. Rev. E 74 (Nov, 2006) 051113.

[14] J. Fingberg, U. M. Heller, and F. Karsch Nucl. Phys. B392 (1993) 493-517, [hep-lat/9208012].

[15] J. Langelage and O. Philipsen JHEP 01 (2010) 089, [0911.2577]. 